\title{
Effect of data density, scan angle, and flying height on the accuracy of building extraction using LiDAR data
}

\author{
BHARAT LOHANI* and RAJNEESH SINGH \\ Department of Civil Engineering, Indian Institute of Technology Kanpur, \\ Kanpur, India 208016
}

(Received ???; in final form ????)

\begin{abstract}
A Hough transform based approach for extraction of buildings using LiDAR data is presented. It is argued that LiDAR data should be smoothed and sparsed prior to Hough transform for better result. Algorithms to realize this are presented. Further, an algorithm which fits a vector model to extracted buildings is outlined. Simulated LiDAR data have been used to investigate the effect of three parameters (data density, flying height, and scan angle) on the quality of buildings extracted. A set of accuracy indices is proposed for this purpose. It is shown that the data density is the most significant parameter affecting the accuracy of building identification.
\end{abstract}

Keywords: Building identification; Hough transform; LiDAR; LiDAR simulator

\section{Introduction}

The significance of 3D geo-information is increasing day by day with new applications being found. Accurate 3D geo-information can help to make important decisions like placement of telecommunication towers, planning of disaster management, land tax evaluation, etc. It also helps in various modelling studies like noise simulation, heat and exhaust studies, pollutant movement, etc. The 3D details of buildings are an important item of geo-information and can be obtained from land surveys, through photogramme-try (aerial or satellite stereo images) or from LiDAR (Light Detection And Ranging) data.

Till recently, building extraction was being performed mainly by photogrammetry. However, generation of a digital surface model (DSM) and extraction of buildings using photogrammetry is resource and time consuming. In some cases it may even become impossible to obtain DSM due to high object density, occlusions and object complexity in city areas (Guo and Yasuoka 2002).

The advent of LiDAR technology has solved the problem of obtaining DSM as it directly provides $3 \mathrm{D}$ coordinates of the ground points. The points provided by laser scanner are dense and accurate. To utilize this data there is a need to develop algorithms to efficiently and accurately extract information from a large volume of data.

*Corresponding author. Email: blohani@iitk.ac.in

Geocarto International

ISSN 1010-6049 print/ISSN 1752-0762 online (C) 2007 Taylor \& Francis

http://www.tandf.co.uk/journals

DOI: $10.1080 / 10106040701207100$ 
Various approaches have been employed for extracting buildings from LiDAR data. Classification of LiDAR data using remote sensing tools has been investigated by Arefi et al. (2003). They generated normalized difference images and also used reflectance data for their classification approach. Researchers have used several different methods for LiDAR data classification, like height segmentation (Maas 1999), texture based segmentation (Tan and Shibasaki 2002), reasoning on image segments (Zhan et al. 2002), morphological processing, mathematical model fitting (Vu and Tokunaga 2002), classification algorithms (Maas 1999, Voegtle and Steinle 2003, Elberink and Maas 2000) etc. Various methods have been combined to increase the efficiency of classification. Both the first and last return data have been used to get better accuracy (Voegtle and Steinle 2003). Integration of LiDAR data with different data types, like multispectral images (Sohn and Dowman 2003), reflectance information from LiDAR (Elberink and Maas 2000), GIS (geographical information system) maps (Tan and Shibasaki 2002), etc. has been attempted to improve classification accuracy.

This paper concentrates on building extraction using the Hough transform, which has emerged as a valuable technique. The term 'building extraction' encompasses two processes - first, building identification, which is detection of LiDAR points that belong to a building, and second the model fitting (also referred to in the literature as building reconstruction), in which the identified building surfaces are modelled on a vector framework. Vosselman and Dijkman (2001) have used a 3D Hough transform to detect planes in LiDAR data. Hofmann et al. (2003) have made a comparison between 2D and 3D Hough transforms for detecting building planes in LiDAR data. The Hough transform can be implemented using a cluster space described by two slope parameters $\left(s_{x}\right.$ and $s_{y}$ ) and a distance parameter $(d)$ (Maas and Vosselman 1999):

$$
Z=s_{x} X+s_{y} Y+d
$$

The basic element adopted for computing the aforesaid parameters is a triangle. Triangles are generated using a tessellation scheme on LiDAR data. Due to random errors in the data (particularly in the $Z$ direction) the parameters of these triangular elements vary substantially even if the triangles come from the same roof plane. This variation of parameters can be taken care of by clustering of data (i.e. having higher values of quantization in Hough room). However, in case of high data density, on its own this measure may not be able to account for the variation in parameters of triangular planes due to random error. So preprocessing of LiDAR data by smoothing, sparsing, or both, prior to the Hough transform, is desired. Further, how building extraction is dependent upon data characteristics is not known. However, this is fundamental for optimal use of LiDAR data. Finally, in the absence of a proper accuracy analysis procedure, it is difficult to compare the performance of different algorithms or the effect of data characteristics. The aforesaid issues have been either partly explored or not explored in the existing literature, and are the motivation for this work.

\section{Data used}

The availability of a large number of LiDAR data sets with varied specifications is fundamental to this study. However, acquiring this data from the field is not feasible 
considering the cost involved. Furthermore, for the accuracy analysis studies it is desirable that full and accurate ground truth is available. Considering the above, the data used in this paper are generated using a LiDAR simulator (Lohani et al. 2006). In the simulator the terrain (ground surface and buildings on it) is represented using mathematical equations. Mathematical models are also employed to simulate the aircraft trajectory and scanning mechanism. A laser vector, for each transmitted pulse, is computed using the trajectory coordinate, attitude parameters, and instantaneous scan angle. The intersection of this vector with the terrain (i.e. equations representing ground and buildings) gives the coordinate of the LiDAR point. The simulator can also generate LiDAR data with specified errors.

It is imperative that the data density should play a crucial role in the quality of buildings extracted. However, it is not known how the quality would vary for different data densities. Furthermore, the same data density can be achieved for different flying heights or for different scan angles or a combination of both by changing the firing frequency accordingly. With the change in flying height and scan angle, the pattern of spread of data points on the terrain changes. It is interesting to investigate how this impacts the building extraction.

In view of the above discussion, three parameters, viz. data density, scan angle, and flying height are chosen to characterize a data set. A total of 45 data sets (consisting of 11 building roof planes) are generated for different settings of these parameters. These buildings stand on flat ground. No trees were included in simulated data as the aim is to study building extraction accuracy. Further, the presence of trees will only increase the extra lap error, as discussed in the accuracy analysis section later. For a simulated data set the flying height could be any of $500 \mathrm{~m}, 1000 \mathrm{~m}$, and $1500 \mathrm{~m}$ while the scan angle could be any of $10^{\circ}, 20^{\circ}$, and $30^{\circ}$. Further, the data density could be any of $1,2,4,8,12$ points $/ \mathrm{m}^{2}$. To achieve the desired data density for fixed scan angle and flying height the firing frequency is varied accordingly. A random error with standard deviation of $0.45 \mathrm{~m}$ in the $X$ and $Y$ coordinates and $0.15 \mathrm{~m}$ in the $Z$ coordinate is also added to the simulated data. It is understood that the quantum of random error depends upon the altitude and the location of LiDAR point on swath. However, this is maintained the same here for all date sets as the main emphasis is to study the effect of the chosen parameters and not that of varying random errors. The simulated data mimic cartesian coordinates on UTM projection with WGS84 datum.

\section{Methodology for building extraction}

The methodology for building extraction is divided into two parts. First part consists of identification of group of points corresponding to a roof plane by using Hough transform. The second part consists of fitting a model to the points within a group. The following paragraphs describe these steps. Matlab is used for coding the algorithm.

\subsection{Detection of building roof points}

Building roof points are detected using the Hough transform. The methodology adopted is shown in figure 1. LiDAR data in WGS84 are normalized by transforming the coordinates of points to the local origin (mean $x$, mean $y$, minimum $z$ ). This helps to reduce the $Z$ intercept parameter $(d)$ of the plane equation 


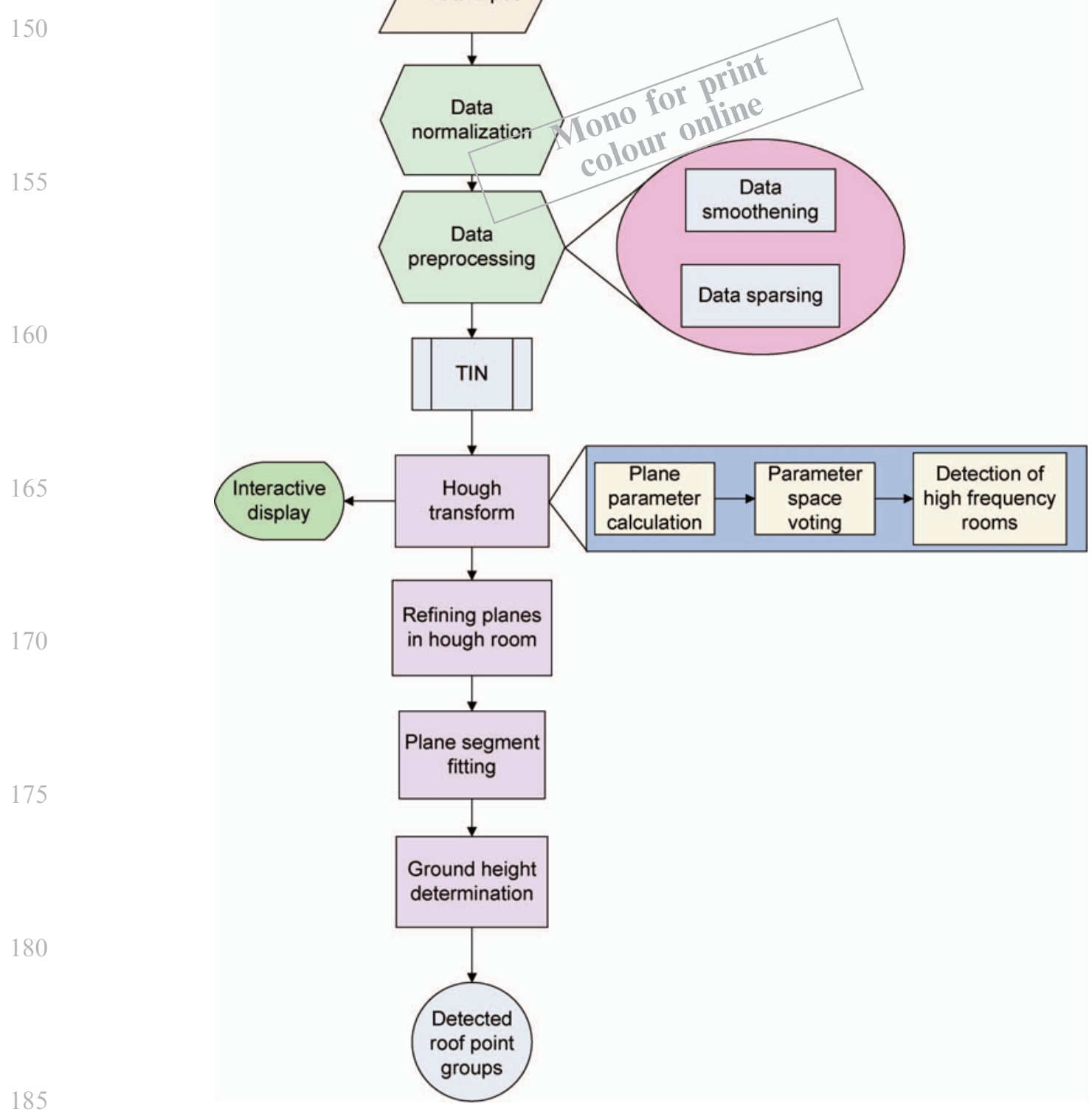

Figure 1. Methodology for identification of group of data points belonging to a roof plane.

$Z=s_{x} X+s_{y} Y+d$. This parameter is further constrained in range by specifying the maximum slope of the roofs present in the data. The data can be transformed back to the original coordinates once the building model fitting is over. The point elevation coordinates are smoothed if the data density is lower (up to eight points $/ \mathrm{m}^{2}$ ) while for higher data density both sparsing and smoothing are done.

Each data point is evaluated for smoothing. A plane is fitted to the point under consideration and its neighbouring points (figure 2). The standard deviation of the 


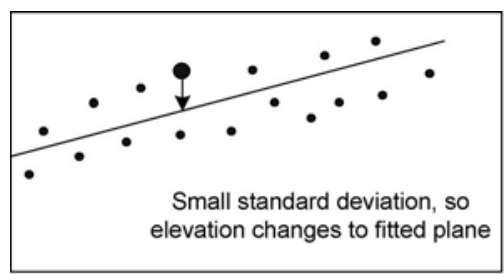

(a)

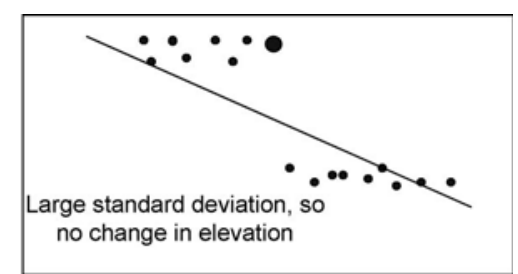

(b)

Figure 2. Smoothing raw LiDAR data to minimise the effect of random error. The dots are LiDAR data while the line represents the plane fitted. Larger dot is the point under consideration. (a) Standard deviation of separation less, so elevation changes to that of fitted plane; (b) Large standard deviation of separation, so no change in elevation for this point on edge.

separation of these points from the fitted plane is computed in the $z$ direction. If the standard deviation is less than the standard deviation of the data error the elevation of the point under consideration is replaced by the corresponding elevation on the fitted plane. This ensures that the data are smoothed for random error while preserving the edges. Sparsing is done by removing the neighbours of a point which are within a specified distance from the point. Data points which are on edges, determined using the edge detector on the LiDAR image, and which were removed in the process of sparsing, are added back. The sparse data produce larger basic computation elements, i.e. triangles, thus minimizing the effect of random error on slope parameters computed.

Data resulting from the above step are triangulated in plan by using a $2 \mathrm{D}$ Delaunay triangulation. Using the coordinates of vertices of triangles the plane parameters, i.e. $s_{x}, s_{y}$, and $d$ are computed for each triangle. Hough room quantization is done based on the specified quantization levels for different parameters. These quantized levels facilitate voting to parameter space. The Hough room cells with high frequency of votes are further analysed. All the triangles with similar plane parameters vote in the same cell. However, all these may not belong to one roof plane. So different point groups in one cell need to be separated. This is realized by gridding all the points of a cell to a binary image with grid size being equal to the average data spacing (figure 3). Grid elements where a data point is present are made TRUE while the rests become FALSE. A morphological closing operator (with a $3 \times 3$ structuring element) is operated on the resulting binary image to close the holes in the data group. All the elements of an eight-connected point group are given a unique ID using an indexing algorithm. The IDs are mapped back to the points in the Hough room. The point groups, having area and number of points larger than the specified thresholds, are kept for further analysis.

Following the above, a segmentation based fitting is carried out to collect all the points belonging to one roof in one point group. This is done by starting with a point group and fitting a plane to it by least squares. The perpendicular distance of neighbouring points (within a distance threshold) to the fitted plane is calculated. If the point is within $3 \sigma$ of the elevation error it is included in point group and the plane parameters are updated. This is done till no other point is being included into the group. This step helps to merge groups having different IDs but belonging to the same roof. 


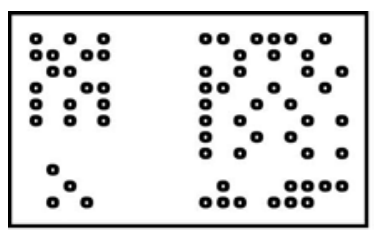

(a)

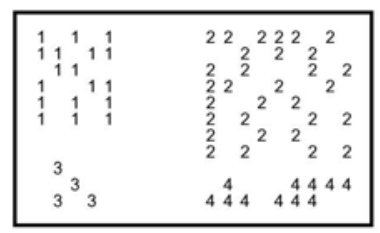

(d)

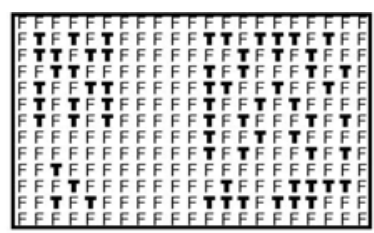

(b)

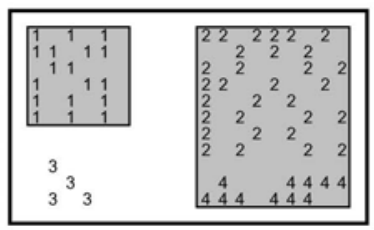

(e)

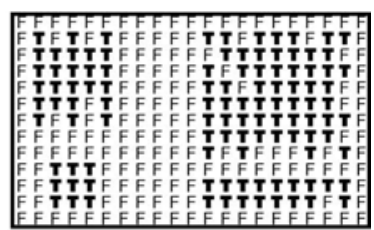

(c)

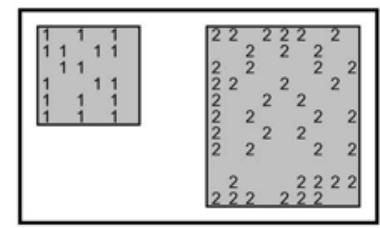

(f)

Figure 3. Segmentation of one cell of hough room. (a) Raw data; (b) Gridding of raw data with TRUE (T) and FALSE (F) pixels; (c) Morphological closing on grid to fill holes; (d) ID assigned to each individual group; (e) Groups merging; (f) Retention of only major groups. (The figure is only indicative of processing steps and not an exact graphical representation.)

\subsection{Building model fitting}

LiDAR points identified corresponding to building roofs in the above step are represented using a vector framework. This is realized by fitting a plane to the point group by least squares and finding the edges of the point group. The steps used for building model fitting are shown in figure 4 . For detection of the edge points the roof point group is converted to binary raster with a pixel size equal to the average data spacing (figure 5). Holes in the raster data are filled by morphological closing. Boundary pixels are determined using the bwboundaries() function of the Matlab software. The roof group points are mapped onto the raster and the points corresponding to edge pixels are selected for further fitting of line by contour approximation and orthogonalization. An algorithm, similar in concept to (Douglas and Peucker 1973), but specifically adapted to identify roof edges, has been developed for this purpose.

In this, two points having maximum separation in the boundaries are flagged as control points. The distance of other points is calculated from the line formed by joining the control points (figure 6(a)). The point at maximum distance from this line is again flagged as a control point. Consideration of the direction of the contour ensures that the points only on one side of the line are evaluated for selection. Again the distance of points is calculated from the lines formed. The point at maximum distance from its nearest contour line is made a control point if its distance from the contour line is greater than a threshold distance. This is done till all the points are within the threshold distance (half the minimum side length specified for building) from the nearest contour line (figure 6(c)). Then lines are fitted to all points between two consecutive control points. The longest line is taken as the main orientation of building and other lines are made orthogonal to that line. The lines obtained are later intersected to get the corners of the roofs.

Once the building roofs are detected the roofs which are close (within a threshold) to each other are intersected to get the line of intersection of roofs. The next step is to 


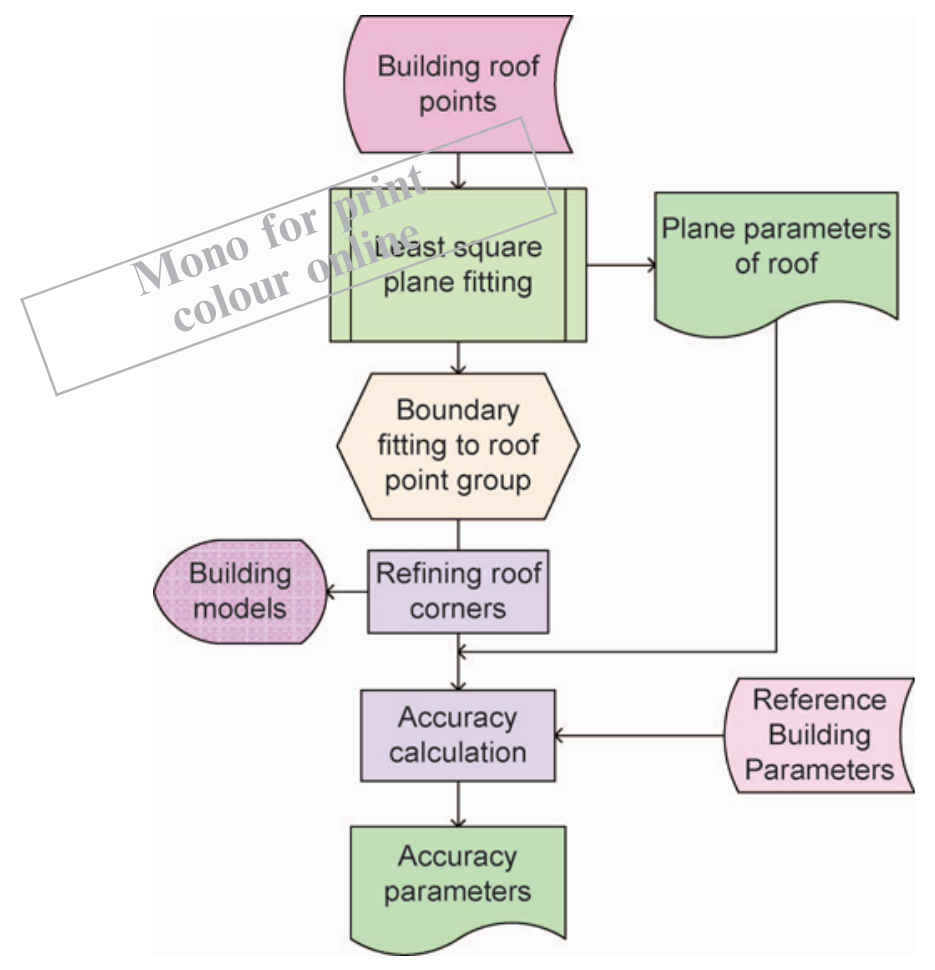

Figure 4. Steps of building model fitting and accuracy analysis.

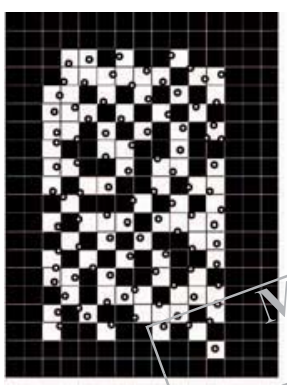

(a)

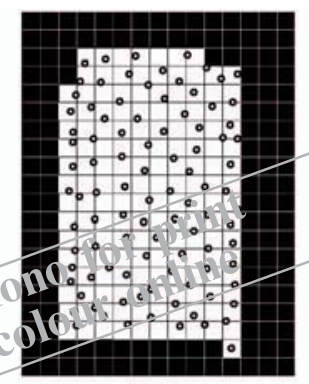

(b)

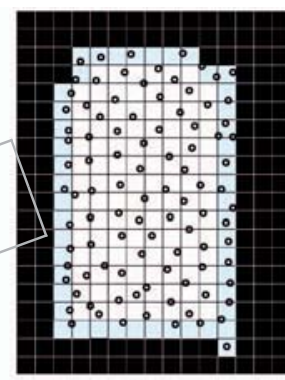

(c)

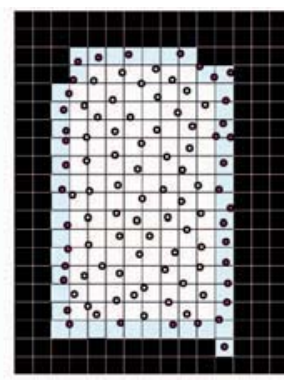

(d)

Figure 5. Schema of edge point detection of roofs. (a) Gridding roof points; (b) Morphological Closing on grid to fill holes; (c) Application of Bwboundaries() function; (d)Edge points detected.

find the height of the point group above ground. This is accomplished based on the difference of the elevation of edge point group and their neighbours on the ground. The point group height helps to differentiate roofs from the planar surfaces formed due to flat ground. Using the corner coordinates of the roofs the building models can be plotted. 


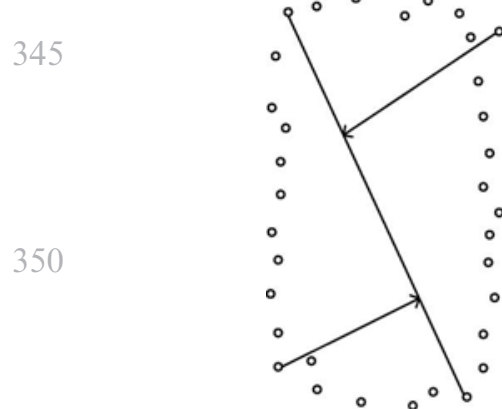

355

(a)

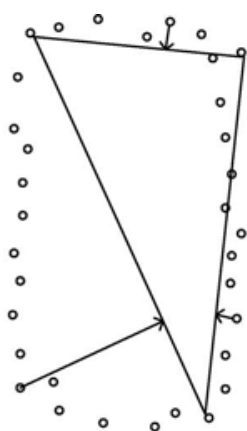

(b)

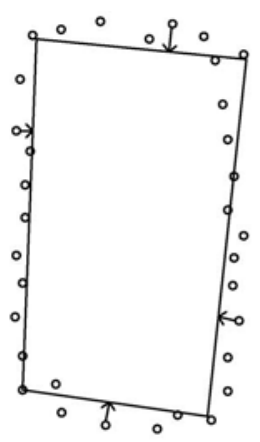

(c)

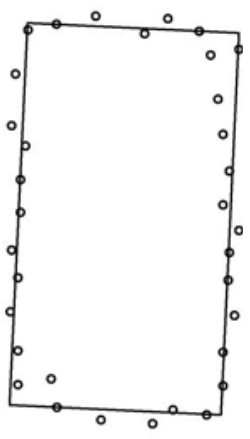

(d)

Figure 6. Schema of line fitting to edge points by contour approximation and orthogonalisation. (a) Maximum distance point from line; (b) Joining point at maximum distance; (c) Contour approximation of edge points; (d) Resultant boundary of Line fitting and orthogonalisation.

\section{Development of accuracy indices}

In order to assess the quality of buildings extracted the ground truth and a method for comparison are required. A set of accuracy measures is proposed below. In the existing literature the accuracy analysis is carried out mostly by employing the areal measures. However, inclusion of the corner distance, slope and perimeter indices makes error analysis more comprehensive. Only areal measures lead to misleading conclusions on some occasions.

- Corner distance: Mean value of distance between corresponding nearest corner of extracted and reference building:

$$
\mathrm{Cde}=\frac{\sum_{i=1}^{n} \operatorname{dist}\left(P_{i}^{\mathrm{ref}}, P_{i}^{\mathrm{ext}}\right)}{n}
$$

where Cde is the corner distance accuracy of a building, $P_{i}^{\text {ext }}$ and $P_{i}^{\text {ref }}$ are the $i^{\text {th }}$ corner points of the identified and reference buildings, respectively, $\operatorname{dist}(A, B)$ is a function which calculates the distance between points $A$ and $B$, and $n$ is the number of corners of the building.

- Slope accuracy: This is the difference of the absolute values of the reference building slope and the actual slope detected:

$$
S D_{i}=\left|S l_{\mathrm{ref}}\right|_{i}-\left|S l_{\mathrm{ext}}\right|_{i}
$$

where $S D_{i}$ is the slope difference, $S l_{\text {ref }}$ and $S l_{\text {ext }}$ are the slopes of the reference and extracted buildings, respectively, and $i$ represents the direction of the slope $\left(S_{x}\right.$ or $S_{y}$ direction).

- Area difference: This is calculated using the formula:

$$
\text { Area difference }=\frac{\text { Area }\left(\text { Poly }_{\text {ref }}\right)-\text { Area }\left(\text { Poly }_{\text {ext }}\right)}{\text { Area }\left(\text { Poly }_{\text {ref }}\right)}
$$


where $\operatorname{Area}\left(\operatorname{Poly}_{x}\right)$ is the area calculation function for calculation of the area of Poly $y_{x}$ polygon. Poly ref is the reference building polygon and Poly $\mathrm{y}_{\mathrm{ext}}$ is the extracted building polygon.

- Perimeter difference: This is calculated using the formula:

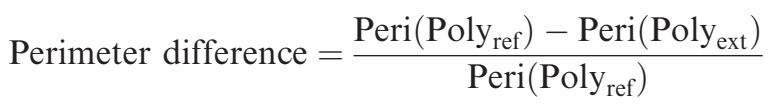

where Peri $\left(\operatorname{Poly}_{x}\right)$ is the perimeter calculation function for Poly $x$. Other terms are as defined above.

- Area Overlap: This index is calculated using the formula:

$$
\text { Area overlap }=\frac{\text { Area }\left(\text { Poly }_{\text {ref }} \cap \text { Poly }_{\text {ext }}\right)}{\text { Area }\left(\text { Poly }_{\text {ref }}\right)}
$$

where all the terms are defined as above.

- Area extralap: This is calculated as:

$$
\text { Area extralap }=\frac{\text { Area }\left(\text { Poly }_{\text {ext }}-\text { Poly }_{\text {ref }}\right)}{\text { Area }\left(\text { Poly }_{\text {ref }}\right)}
$$

The corner distance and slope accuracies are reported in metres and degrees, respectively, while the rest of the indices are unitless. Further, the aforesaid indices are defined for an individual building. To define the accuracy for all buildings together in a data set, the accuracy indices of individual buildings are averaged. The standard error of mean is also computed.

\section{Result and discussion}

All 45 data sets are processed by the building extraction algorithm. The quantization for $S_{x}$ and $S_{y}$ is kept at $7^{\circ}$ and $2 \mathrm{~m}$ for the $z$ intercept. The result shown in figure 7 is for the simulated data obtained from an altitude of $500 \mathrm{~m}$ with scan angle $20^{\circ}$ and a data density of 8 points $/ \mathrm{m}^{2}$. The algorithm was also tested on actual data with satisfactory performance. The results of same are not included in this paper as the main focus of this paper is on simulated data. The building models generated from 45 data sets are compared with their truth (the parameters of buildings used for simulating the data) using the accuracy indices proposed above. In most of the cases there are enough data points to yield statistically significant results. For example, the corner distance value of the plot in figure 9(a) is an average of 660 corner distances (four corners $X, 11$ roof planes $X, 15$ data sets). The extracted building models (shown in figure 7) and their accuracy analysis indicate satisfactory performance of the proposed method.

The results of an accuracy analysis are presented in two formats. In the first case, the results presented are for all data generated with $10^{\circ}$ scan angle (figure 8 ). The ordinate scale values in these plots are set to accommodate the maximum error value. The corresponding standard error is also plotted. These results show how 


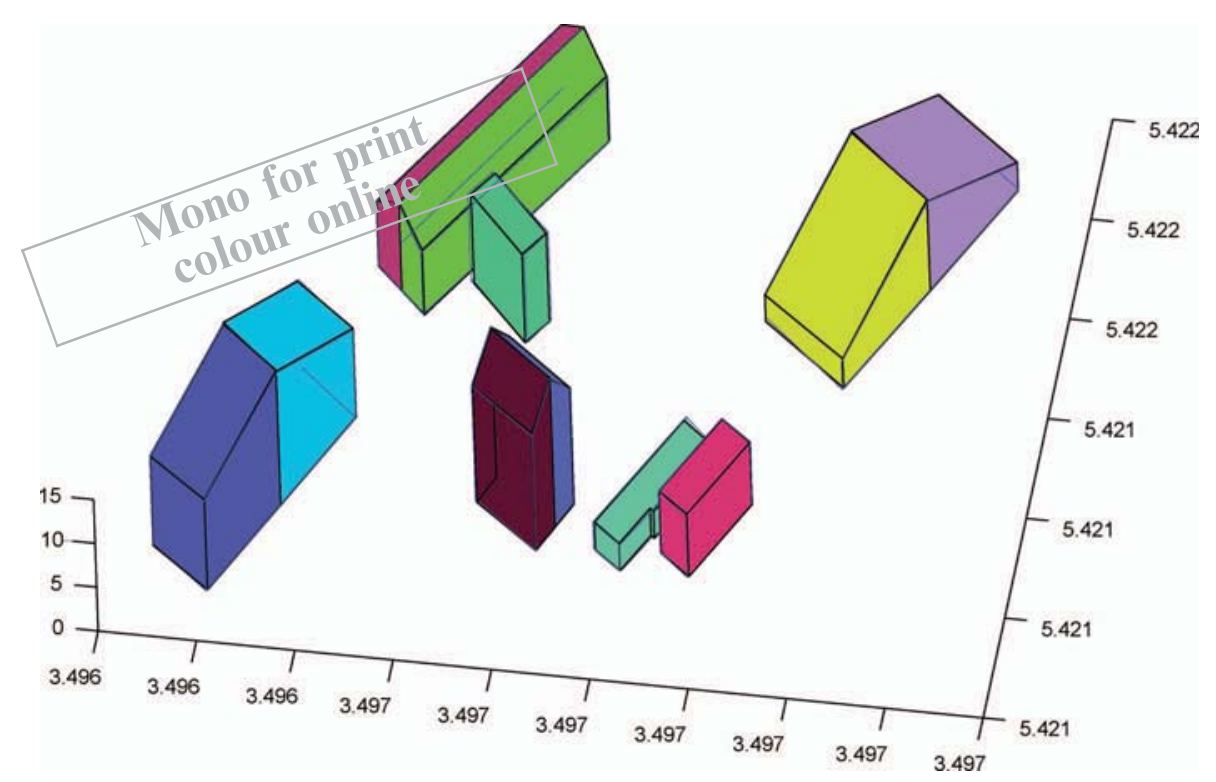

Figure 7. Final building models for simulated data. Shades are used only to distinguish different planes. Easting and Northing in $10^{6} \mathrm{~m}$ units. Altitude in $\mathrm{m}$ units.

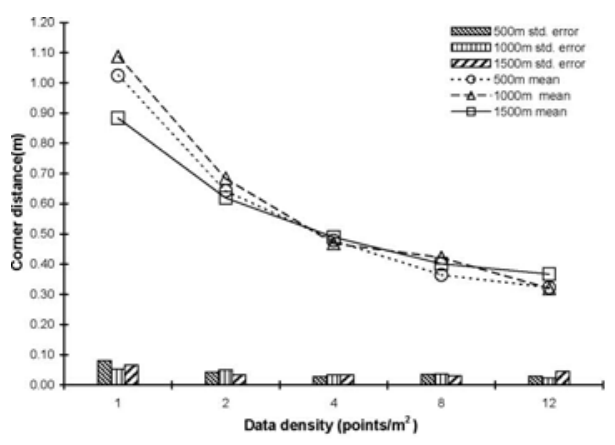

(a) Corner distance

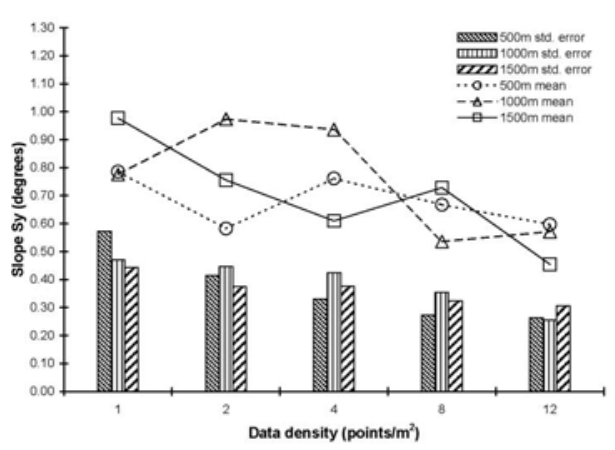

(c) Y direction slope error

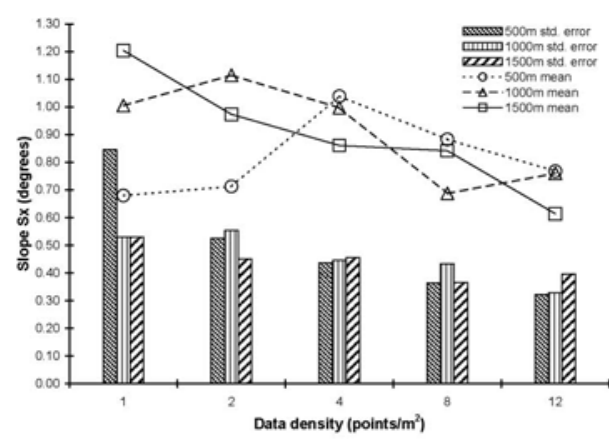

(b) $\mathrm{X}$ direction slope error

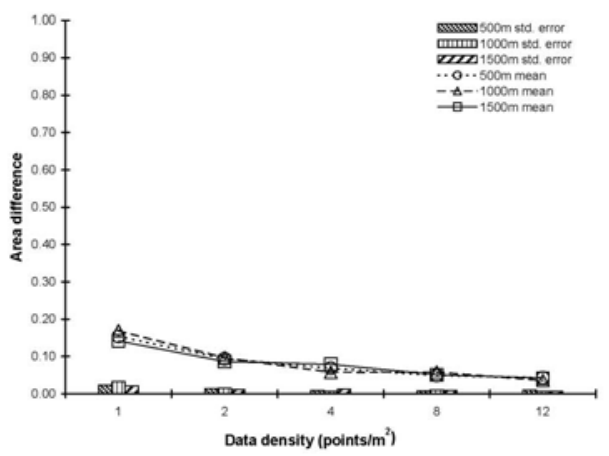

(d) Area difference

Figure 8. Variation of accuracy indices for $\pm 10^{\circ}$ scan angle with flying height and data density. 


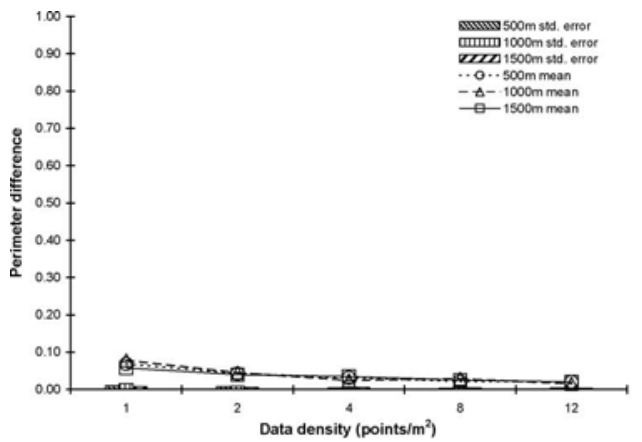

(e) Perimeter difference

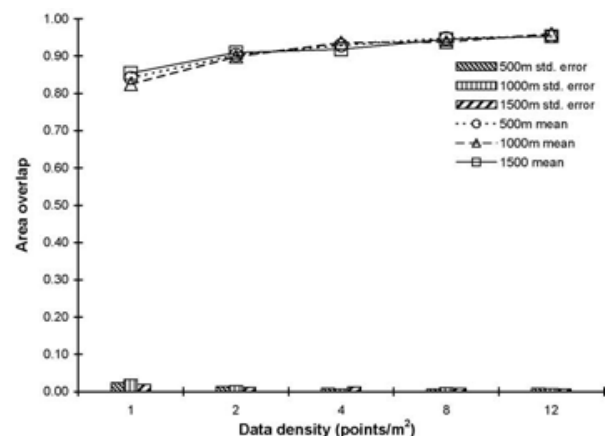

(f) Area overlap

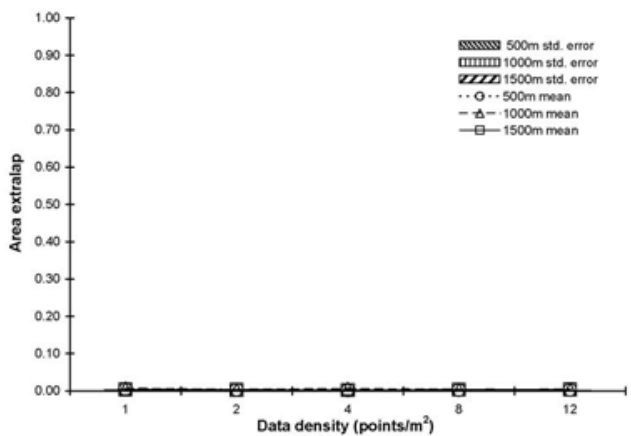

(g) Area extra lap

Figure 8. (Continued).

accuracy varies with changes in data density and height for a constant scan angle. The results for other scan angles are similar, thus are not shown here. In the second case, the results are averaged all across the data. For example, to know how the corner distance accuracy varies with height, all data obtained from a height with different data densities and scan angles are averaged. These overall results are presented in figure 9. This result attempts to explain, despite the variation in other parameters (e.g. data density and scan angle), how the accuracy is being affected by the parameter under consideration (e.g. flying height). Here the ordinate axis scale is set between 0.00 and 1.00 . The mean errors computed for all accuracy indices fall within this range. Moreover, for corner distance and slope errors this axis represents units in metres and degrees, respectively, while for other indices it indicates the unitless value of ratio.

The mean values and standard errors of the mean plotted in figure 8(a) exhibit significant improvement in corner distance accuracy with increase of data density (e.g. 1 to 8 points $/ \mathrm{m}^{2}$ ). However, the improvement from 8 to 12 points $/ \mathrm{m}^{2}$ is not significant. Further, there is no significant systematic variation of accuracy for data obtained from different flying heights. Similar behaviour is observed for all other indices. The near-zero values of extralap are because the algorithm does not jump out of building roof planes and also because there are no adjoining trees, etc. in the simulated data. 


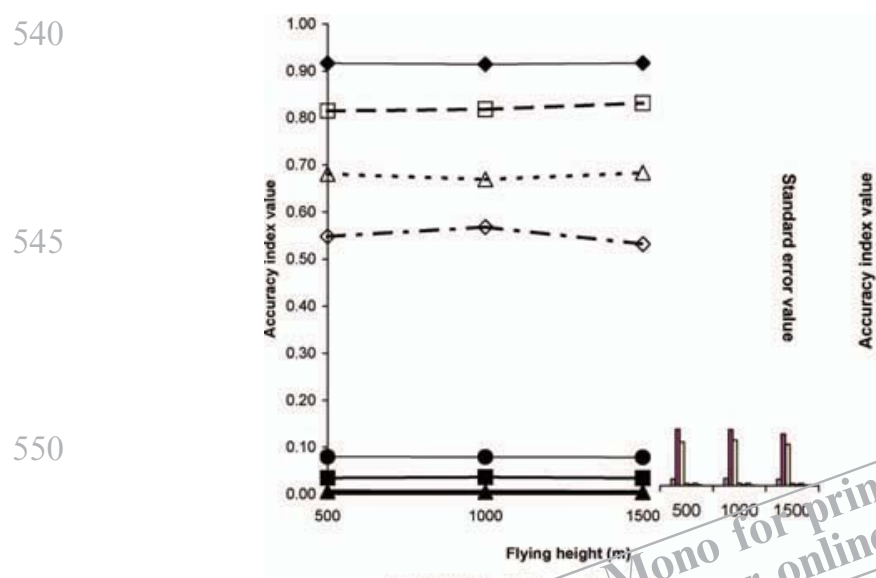

(a) With filying height

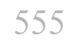

560

565

570

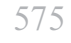

580

585

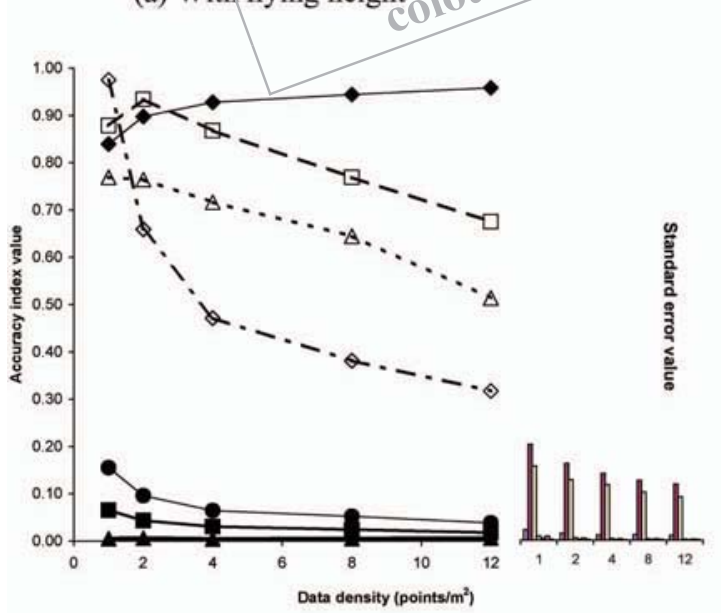

(c) With data density

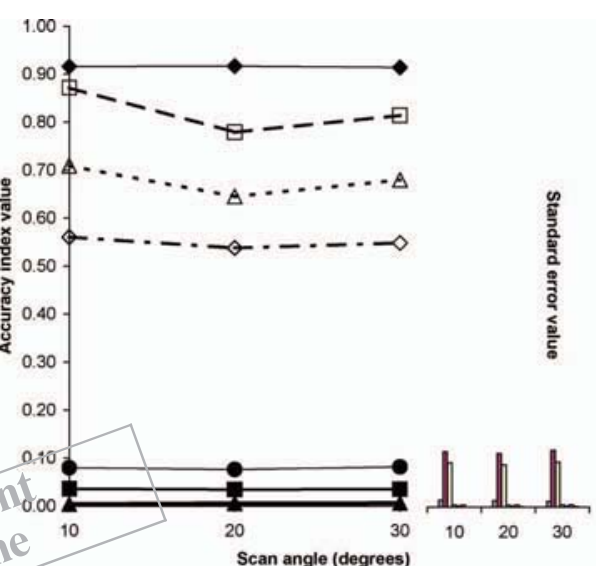

(b) With scan angle

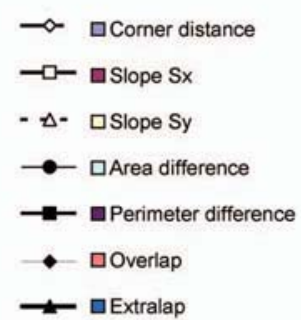

(d) Legend for mean and standard error

Figure 9. Variation of accuracy indices and their standard error for the variation in one parameter and irrespective of values of other parameters.

The results in figure 9 show that the flying height and scan angle variation do not affect building identification. However, as seen in figure 9(c) all accuracies improve with data density. Except for the slope, the standard errors for other measures are significantly small, thus showing the statistical significance of the results.

From figures 8 and 9 it is clear that with an increase in data density the corner distance accuracy improves most. This is due to the fact that building corner identification depends mainly on the definition of roof edges. The edges will be located better if more data are available for line fitting. There is a similar reason for the increase in slope accuracy as the roof plane is being defined better with more accurate roof corners. The large standard error for slope indices is because of the smaller buildings used here. A minor change in corner coordinates gives a large rotation to the determined roof planes. For large buildings this error should have smaller standard error. For the same reason, there is a non-monotonic improvement in slope accuracy indices with increase in data density and the standard error is still 
larger when all results are clubbed together (figure 9(c)), though there is monotonic improvement in slope accuracy.

Based on the result of the accuracy analysis for different data densities it is clear that the main factor affecting the accuracy of building identification is data density. The other factors do not affect the performance of building extraction in any systematic manner.

\section{Conclusion}

The Hough transform based approach with data smoothing and sparsing to reduce the effect of random error is effective in extracting buildings accurately. The building modelling algorithm works satisfactorily and produces a vector model of buildings for display and other purposes. However, extraction of edge points of building needs further improvement. From accuracy analysis it is clear that building extraction accuracy mainly depends on data density and there is generally no significant improvement after 8 points $/ \mathrm{m}^{2}$. The flying height and scan angle variations do not affect the accuracy. It is expected that, like the Hough transform method, other approaches to building extraction should also mainly depend on data density, with a similar trend.

\section{References}

Arefi, H., Hahn, M., and Lindenberger, J., 2003, Lidar data classification with remote sensing tools. ISPRS Commission IV Joint Workshop on Challenges in Geospatial Analysis, Integration and Visualization II, Georgia, October 29-31, 2001.

Douglas, D. and Peucker, T., 1973, Algorithms for the reduction of the number of points required to represent a digitized line or its caricature. The Canadian Cartographer, $\mathbf{1 0}$ $112-122$.

ElberinK, S.O. and MAAS, H.-G., 2000, The use of anisotropic height texture measures for the segmentation of airborne laser scanner data. IAPRS, Amsterdam, Vol. XXXIII.

GuO, T. and YASUOKA, Y., 2002, Snake based approach for building extraction from high resolution satellite images and height data in urban areas. 23rd Asian Conference on Remote Sensing, Nepal, November 25-29, 2002.

Hofmann, A.D., MaAs, H.-G., and Streilein, A., 2003, Derivation of roof types by cluster analysis in parameter spaces of airborne laserscanner point clouds. Proceedings of the ISPRS Working Group III/3 Workshop on 3-D Reconstruction from Airborne Laserscanner and InSAR Data, Dresden, October 8-10, 2003.

Lohani, B., Reddy, P.P., and Mishra, R.K., 2003, Airborne Altimetric LiDAR Simulator: An education tool. International Archives of the Photogrammetry, Remote Sensing and Spatial Information Science, Tokyo, Volume XXXVI, Part 6, Tokyo, June 26-30, 2006.

MAAS, H.-G., 1999, Fast determination of parametric house models from dense airborne laserscanner data. IAPRS International Workshop on Mobile Mapping Technology, Bangkok, April 21-23, 1999, 32(2W1).

Maas, H.-G. and Vosselman, G., 1999, Two algorithms for extracting building models from raw laser altimetry data. ISPRS Journal of Photogrammetry and Remote Sensing, 54, $153-163$.

Sohn, G. and Dowman, I., 2003, Building extraction using lidar dems and ikonos images. Proceedings of the ISPRS Working Group III/3 Workshop on 3-D Reconstruction from Airborne Laserscanner and InSAR Data, Dresden, October 8-10, 2003. 
TAN, G. and Shibasaki, R., 2002, A research for the extraction of 3D urban building by using airborne laser scanner data. 23rd Asian Conference on Remote Sensing, Nepal, November 25-29, 2002.

Voegtle, T. and Steinle, E., 2003, On the quality of object classification and automated building modeling based on laser scanning data. ISPRS, XXXIV(3/W13).

Vosselman, G. and Dijkman, S., 2001, 3D building model reconstruction from point clouds and ground plans. Proceedings of the ISPRS workshop on Land Surface Mapping and Characterization Using Laser Altimetry, Annapolis, Maryland, 22-24 October 2001. The International Archives of the Photogrammetry, Remote Sensing and Spatial Information Sciences, Vol. XXXIV part 3/W4 Commission III., pp. 37-44.

Vu, T.T. and Tokunaga, M., 2002, Designing of wavelet-based processing system for airborne laser scanner segmentation. Proceedings of the International Archives of Photogrammetry, Remote Sensing and Spatial Information Science, XXXIV (5/W3).

Zhan, Q., MolenaAr, M., and Tempfli, K., 2002, Building extraction from laser data by reasoning on image segments in elevation slices. In PCV02, page B: 305. 